\title{
Calculation of the dynamics of surface melting during laser annealing
}

\section{Citation}

Surko, C. M., A. L. Simons, D. H. Auston, J. A. Golovchenko, R. E. Slusher, and T. N. C.

Venkatesan. 1979. Calculation of the Dynamics of Surface Melting During Laser Annealing. Applied Physics Letters 34, no. 10: 635-637. doi:10.1063/1.90619.

\section{Published Version}

doi:10.1063/1.90619

\section{Permanent link}

http://nrs.harvard.edu/urn-3:HUL.InstRepos:29407058

\section{Terms of Use}

This article was downloaded from Harvard University's DASH repository, and is made available under the terms and conditions applicable to Other Posted Material, as set forth at http:// nrs.harvard.edu/urn-3:HUL.InstRepos:dash.current.terms-of-use\#LAA

\section{Share Your Story}

The Harvard community has made this article openly available.

Please share how this access benefits you. Submit a story.

\section{Accessibility}




\title{
Calculation of the dynamics of surface melting during laser annealing
}

\author{
C. M. Surko, A. L. Simons, D. H. Auston, J. A. Golovchenko, and R. E. Slusher
}

Bell Laboratories, Murray Hill, New Jersey 07974

\section{T. N. C. Venkatesan}

Bell Laboratories, Crawford Hill Laboratory, Holmdel, New Jersey 07733

(Received 15 January 1979; accepted for publication 27 February 1979)

\begin{abstract}
We present a thermal transport model to describe the melting and resolidification of semiconductors which is observed to occur during annealing with a pulsed laser. The temperature-dependent properties of both the solid and liquid are included. We compare this calculation with experimental results for the time duration of the melted surface for crystalline $\mathrm{Si}$ and $\mathrm{Ge}$. The temperature of the liquid surface as a function of time is calculated and effects associated with the hot liquid and the vapor are also discussed.
\end{abstract}

PACS numbers: $81.40 . \mathrm{Ef}, 79.20 . \mathrm{Ds}, 82.65 . \mathrm{Jv}, 81.60 . \mathrm{Bn}$

Recently, there has been generated considerable interest in the annealing of semiconductor surfaces by pulsedlaser illumination. 'In this context, we have reported experiments to study the dynamics of the melting of the semiconductor surface during and after laser illumination in situations where annealing is observed to occur. ${ }^{2}$ In this letter we report the results of calculations which describe the dynamics of this physical situation in terms of a thermal transport model. While similar models have been discussed elsewhere, ${ }^{3-5}$ to our knowledge this is the first calculation to include the temperature-dependent thermal and optical properties of both the liquid and solid. These parameters turn out to be crucial, for example, in determining the temperature of the liquid surface. We compare the predictions of this model with measurements of the melt duration in crystalline silicon and germanium when illuminated by a pulsed laser at a wavelength of $0.53 \mu^{2,6}$ The laser wavelength and crystalline, as opposed to implanted, material were chosen, since the optical and thermal properties of the material are more accurately known for this situation. Experimentally, it is found that the duration of the liquid in implanted and crystalline silicon are very similar when illuminated with $0.53-\mu$ light, ${ }^{6}$ and therefore our calculations are directly relevant to laser annealing. We also calculate the temperature of the surface as a function of time and incident pulse energy. We find that at the experimentally observed damage threshold in both $\mathrm{Si}$ and $\mathrm{Ge}$, the calculated vapor pressure is $10 \mathrm{~atm}$ at the surface of the material.

The laser pulse provides a heat flux in the solid and liquid of the form

$$
P_{j}(x, t)=\frac{\alpha_{j} E\left(1-R_{j}\right)}{\sqrt{\pi} \Gamma} \exp \left(-\alpha_{j} x\right) \exp \left(\frac{-t}{\Gamma}\right)^{2},
$$

where the material is in the half-space $x \geqslant 0$, the subscript $j$ is either $s$ or $l$, denoting solid and liquid, respectively, the $R_{j}$ 's are the optical reflectivities, the $\alpha_{j}$ 's are absorption coefficients, $E$ is the incident laser energy per unit area, and we have assumed a Gaussian time dependence of the laser pulse with full width at half-maximum $2(\ln 2)^{1 / 2} \Gamma$. We neglect the transmission of the laser energy through the liquid into the solid since liquid $\mathrm{Si}^{8}$ and $\mathrm{Ge}^{9}$ are metals with absorption lengths $\alpha_{l}^{-1}$ less than $100 \AA$ for all wavelengths between 0.4 and $1 \mu$, and therefore a very thin liquid layer absorbs all the laser energy. The laser beam has a radius at the solid surface greater than $1 \mathrm{~mm}$, which is in all cases large compared to the penetration depth of the heat on the time scale of interest, hence the problem is essentially one dimensional. We seek solutions of the one-dimensional heat equation

$$
\frac{\partial}{\partial x}\left(-K_{j}(T) \frac{\partial T}{\partial x}\right)+C_{j}(T) \frac{\partial T}{\partial t}=P_{j}(x, t)
$$

in the solid and liquid, with the boundary condition

$$
\left.K_{s} \frac{\partial T}{\partial x}\right|_{x_{k}}-\left.K_{l} \frac{\partial T}{\partial x}\right|_{x_{k}}=L \frac{d x_{B}}{d t} .
$$

In Eq. (2), $j$ is either $s$ for solid for solid or $l$ for liquid. In the Eqs. (2) and (3) the $K_{j}$ 's and $C_{j}$ 's are the solid and liquid thermal conductivities and specific heats, respectively, $L$ is the latent heat of melting, and $x_{B}$ is the location of the melt boundary. Equation (2) describes the flow of heat in the liquid and solid, and Eq. (3) describes the melting and resolidification processes. We require $T \rightarrow 300 \mathrm{~K}$ as $x \rightarrow+\infty$ and $\partial T / \partial x=0$ at $x=0$. Since $K_{s}$ is a strong function of temperature varying by a factor of 7 from $300 \mathrm{~K}$ to the melting point in both $\mathrm{Si}$ and $\mathrm{Ge}$, Eq. (2) is intrinsically nonlinear, and we solve the system of Eqs. (1)-(3) numerically on a computer. The values of $R_{s}, R_{l}, \alpha_{l}, \alpha_{s}, C_{s}, C_{l}$, and $K_{s}$ for both silicon and germanium are taken from experimental data. ${ }^{7-12}$ The liquid thermal conductivity $K$, has not been measured experimentally. We calculate its value for both $\mathrm{Si}$ and $\mathrm{Ge}$ using measurements of the electrical conductivity $\sigma_{l}(T)$ and the Weidermann-Franz law which relates $K_{l}$ to $\sigma_{l}$. Data for $\sigma_{l}$ exists over much of the temperature range of interest. ${ }^{13}$ We consider laser pulses with duration of 20-100 nsec FWHM.

The calculations show that the semiconductor surface temperature $T_{s}$ rises rapidly during the laser pulse while the heat begins to diffuse into the solid. As the surface melts, $T_{s}$ continues to increase and the melt boundary $x_{B}$ propagates 
rapidly into solid. After $x_{B}$ reaches its maximum, heat continues to diffuse into the solid and $x_{B}$ decreases to zero over a time which can be as long as several microseconds. Our calculations of $x_{B}(t)$ are qualitatively similar to previous calculations, ${ }^{4,5}$ but details such as the propagation velocity of the melt front and the crystal regrowth velocity are different since they depend sensitively on the assumed values of $\alpha_{l}$ and $K_{l}$ of the liquid metallic state.

Shown in Fig. 1 by the open circles are the experimental measurements ${ }^{6}$ of the time duration $\tau$ of the melted liquid surface for crystalline Si and Ge for incident laser light at a wavelength of $0.53 \mu$. The highest-energy data point corresponds in each case to the threshold for surface damage. The dashed curves represent the calculation for a liqud-state reflectivity of 0.72 for $\mathrm{Si}$ and 0.76 for Ge which corresponds to the measured ${ }^{8}$ liquid-state parameters. The laser pulse for these experiments had a duration of $30 \mathrm{nsec}$ FWHM. The calculations correspond to a duration of $33 \mathrm{nsec}$ FWHM.

The model and experiment disagree by about a factor of 2 in $\tau$ which correspnds to a factor of less than 2 in incident energy $E$. While $\tau$ depends on several parameters, the most sensitive over most of the range shown in Fig. 1 are $K_{s}$ and $R_{l}$. It is difficult to see how $K_{s}$ might be modified, but there are several mechanisms which might lead to a different value of $R_{l}$. Shown in Fig. 1 by the solid curves are the predictions of the present calculation with $R_{l}$ adjusted for best fit in each case. We find $R_{l}=0.57$ for $\mathrm{Si}$ and 0.57 for $\mathrm{Ge}$ also. Shown by

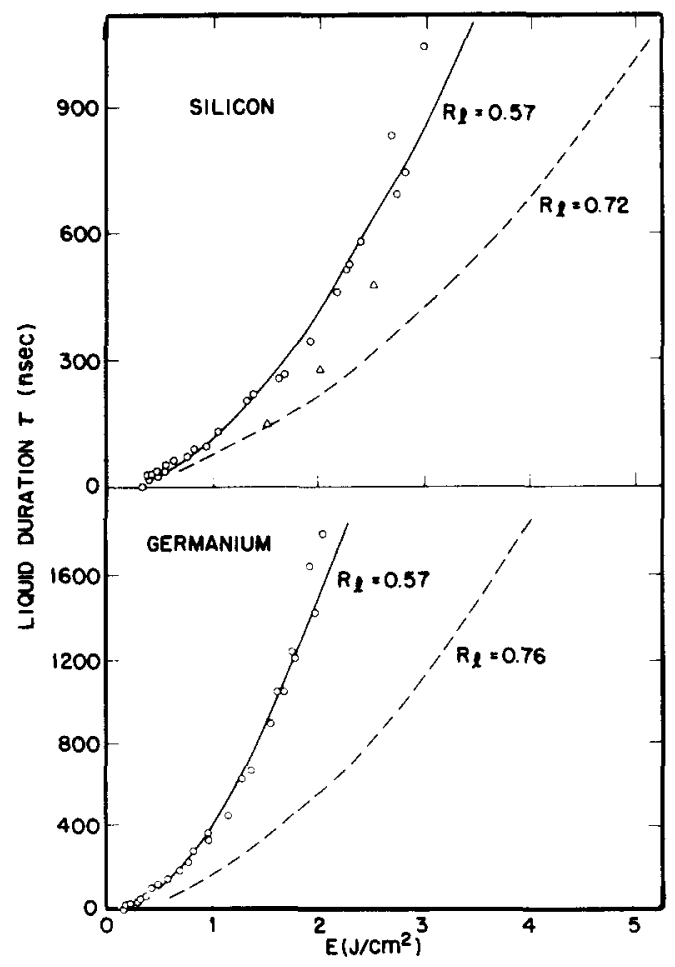

FIG. 1. The duration $\tau$ of the liquid is shown as a function of the incident energy $E$ for both $\mathrm{Si}$ and $\mathrm{Ge}$. The open circles are data from Ref. 6 . The dashed curves are the results of the calculation with previously measured values of $R_{l}$, and the solid curves are the results of the calculation with $R_{l}$ adjusted for best fit to the data. The open triangles are the calculation of Baeri et al. (Ref. 3).

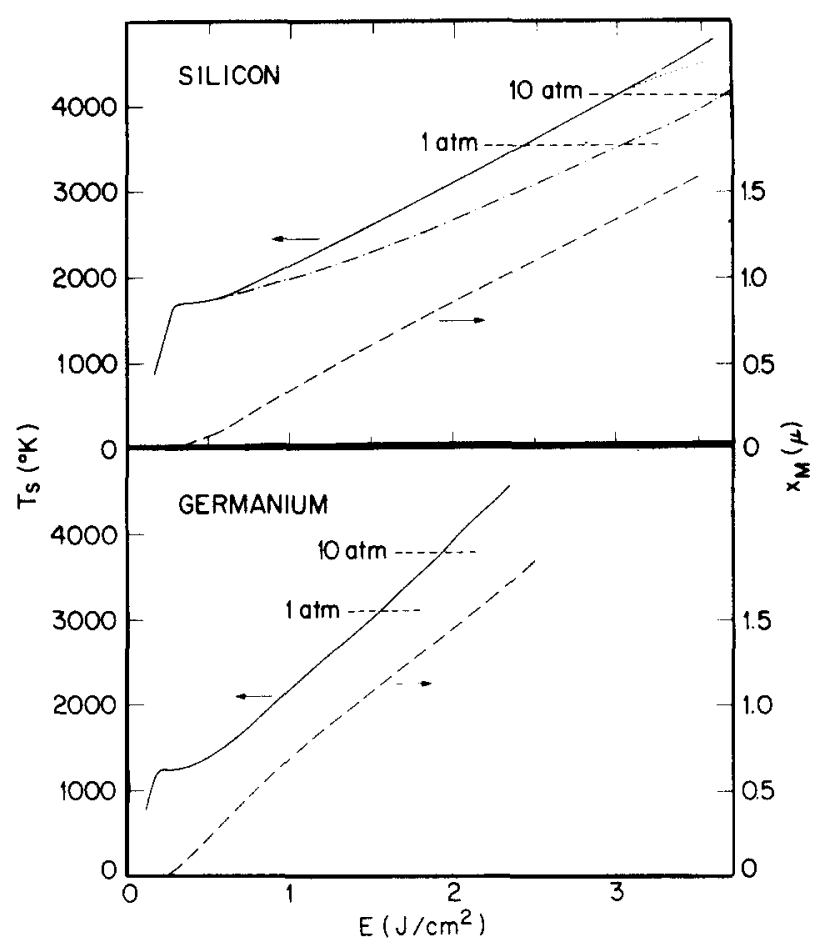

FIG. 2. The maximum surface temperature $T_{s}$ (solid curves) and maximum melt depth $x_{M}$ (dashed curves) as a function of $E$ are calculated for $\mathrm{Si}$ and $\mathrm{Ge}$, assuming $R_{l}$ is 0.57 for both and the laser pulse duration is $33 \mathrm{nsec}$ FHWM. The temperature at which the vapor pressure of material reaches 1 and $10 \mathrm{~atm}$ are marked. Also shown is $T_{s}$ for $\mathrm{Si}$ with a 50-nsec-duration laser pulse (dot-dashed curve) and the effect of including evaporation in $\mathrm{Si}$ (dotted curve).

the open triangles are the calculations of Baeri et al. ${ }^{3}$ for $\mathrm{Si}$ which assumes one value of reflectivity of 0.6 for both liquid and solid Si. This value differs significantly from the measured value ${ }^{8}$ of 0.72 for liquid $\mathrm{Si}$ but is close to the value of 0.57 which we find necessary for best fit to the data. These two calculations therefore agree reasonably well over the range with which we can compare them in Si.

The value of transmission into the liquid phase $\left(1-R_{l}\right)$ necessary for best fit to the data is 1.5 times the previously measured value for $\mathrm{Si}$ and 1.8 times that value for $\mathrm{Ge}^{8,{ }^{89}} \mathrm{Our}$ experimental measurements of the surface reflectivity during the laser pulse indicate that $R_{l}$ is 0.66 at the melting point and decrases to 0.63 at higher incident energies. ${ }^{6}$ Using these values of $R_{l}$, the calculation and experiment are in much better agreement. There are several possibilities for an enhanced coupling of light to the liquid in the situations relevant to laser annealing. The liquid-state temperature may affect the optical constants, which are measured ${ }^{8,9}$ only near melting point, and may be different at higher temperatures. In addition, there is considerable evidence that ripples in the material surface are produced at a wavelength comparable to that of the incident laser. ${ }^{14}$ These ripples may affect the actual liquid-state transmission in a manner similar to that of coupling light to an optical grating.

Shown in Fig. 2 are the predictions of the model for the maximum surface temperature $T_{s}$ and the maximum melt depth $x_{M}$ as a function of incident energy. These calcula- 
tions assume the value of $R_{l}$ of 0.57 for $\mathrm{Si}$ and $\mathrm{Ge}$ which is required for agreement between these calculations and the experimental results in Fig. 1. The temperature at which the vapor pressure reaches 1 and $10 \mathrm{~atm}$ are marked. It is interesting that the experimentally observed energy at which damage occurs corresponds to a vapor pressure of about 10 atm for both $\mathrm{Si}$ and Ge. Damage may be due to plasma breakdown in the vapor of the evaporated material. Evaporation contributes significantly to the power balance only at the highest laser energies as shown by the dotted curve in Fig. 2 which includes evaporative heat loss. Also shown in Fig. 2 for $\mathrm{Si}$ are the results of the calculation of $T_{s}$ for different values of laser pulse duration.

The high temperature of the liquid can enhance the rate of diffusion of impurities such as those introduced in ion implantation. For incident energies $E$ near the threshold for damage, the diffusion coefficient near the surface averaged over the duration of the liquid state is about a factor of 2 greater than the value at the melting. ${ }^{15}$

Another parameter which is calculated is the melt-front velocity, $d x_{B} / d t$, as a function of time. The propagation of the melt into the solid depends on $\alpha_{l}^{-1}$ and $K_{l}$. We find that the maximum value of $d x_{B} / d t$ is $3 \times 10^{3} \mathrm{~cm} / \mathrm{sec}$ for a $33 \mathrm{nsec}$ FWHM laser pulse for values of $E$ just below damage. We note that this is about $10^{-2}$ of the sound velocity, and, consequently, the thermodynamic model is plausible on these time scales. The velocity of return of the melt front to the surface of the material in Si ranges from $600 \mathrm{~cm} / \mathrm{sec}$ for a $0.6-\mathrm{J} / \mathrm{cm}^{2}$ pulse (just above the melting threshold) to $130 \mathrm{~cm} / \mathrm{sec}$ for a $3.0-\mathrm{J} / \mathrm{cm}^{2}$ pulse (near the damage threshold).

We have presented a thermal transport model to de- scribe the dynamics of the melting and resolidification of the material surface observed to occur during laser annealing. We find that the experimentally observed duration of the melted surface in both crystalline $\mathrm{Si}$ and $\mathrm{Ge}$ can be explained by a thermal transport model if one assumes a liquid-state transmission which is a factor of 1.5-1.8 times the values previously measured at the melting point.

We wish to acknowledge the extensive assistance of N.L. Schryer in the computer solution of Eqs. (1)-(3).

'H.R. Leuchtag, Phys. Today 31, 17 (978), and references therein. 'D.H. Auston, C.M. Surko, T.N.C. Venkatesan, R.E. Slusher, and G.A. Golovchenko, Appl. Phys. Lett. 33, 437 (1978).

P. Baeri, S.U. Campisano, G. Foti, and E. Rimini, Appl. Phys. Lett. 33, 137 (1978).

${ }^{4}$ J.C. Wang, T.F. Wood, and P.P. Pronko, Appl. Phys. Lett. 33, 455 (1978).

I.B. Khaibullin, E.I. Shtyrkov, M.M. Zaripov, R.M. Bayzitov, and M.F. Galjanudinov, Radiat. Eff. 36, 225 (1978).

'D.H. Auston, G.A. Golovchenko, A.L. Simons, C.M. Surko, and T.N.C. Venkatesan, Appl. Phys. Lett. (to be published).

W.C. Dash and R. Newman, Phys. Rev. 99, 1151 (1955).

${ }^{8}$ K.M. Shvarev, B.A. Baum, and P.V. Gel'd, Sov. Phys.-Sol. State 16, 2111 (1975).

'J.N. Hodgson, Philos. Mag. 9, 509 (1961).

${ }^{10}$ S.G. Tomlin, E. Khawaja, and G.M.K. Thutupalli, J. Phys. C 9, 4335 (1976).

"Selected Values of Thermodynamic Properties of the Elements, edited by R.T. Hultgren, P.D. Desai, D.T. Hawkins, M. Gleiser, K.K. Kelley, and D.D. Wigwan (American Society for Metals, Metals Park, Ohio, 1973). ${ }^{12}$ C.J. Glasbrenner and G.A. Slack, Phys. Rev. 134, A 1058 (1964)

'V.M. Glazov, S.N. Chizhevskaya, and N.N. Glagoleva, Liquid Semiconductors (Plenum, New York, 1969), Chap. 3.

${ }^{14}$ H.J. Leamy, G.A. Rozgonyi, T.T. Sheng, and G.K. Cellar, Appl. Phys. Lett. 32, 535 (1978), and references therein.

${ }^{15}$ C.M. Surko, A.L. Simons, D.H. Auston, R.E. Slusher, and T.N.C. Venkatesan, Proceedings of the Laser-Solid Interactions Symposium of Materials Research Society, Boston, 1978 (American Physical Society, New York, to be published).

\title{
High optical power density emission from a "window-stripe" AIGaAs double-heterostructure laser
}

\author{
H. Yonezu, I. Sakuma, T. Kamejima, M. Ueno, K. Iwamoto, I. Hino, and I. Hayashi
}

Central Research Laboratories, Nippon Electric Co., Ltd., Takatsu-ku, Kawasaki, Japan

(Received 3 November 1978; accepted for publication 16 February 1979)

\begin{abstract}
Extremely high optical power density emission $\left(10^{7} \mathrm{~W} / \mathrm{cm}^{2}\right)$ was achieved with a new $\mathrm{Zn}$ diffused "window-stripe" laser by eliminating the restriction of the catastrophic optical mirror damage (COMD). The maximum available optical power was at least one order of magnitude higher than the COMD threshold in conventional structures. Furthermore, gradual degradation due to the mirror oxidation has been reduced significantly under $\mathrm{cw}$ operation.
\end{abstract}

PACS numbers: 42.55.Px, 85.60.Jb

The maximum available optical power from semiconductor lasers has been limited by the catastrophic optical mirror damage (COMD), which is a local destruction of laser mirrors at high optical power density emission. ${ }^{i-3}$ The critical optical power density of COMD is typically of the order of $10^{6} \mathrm{~W} / \mathrm{cm}^{2}$ in pulsed operation for AlGaAs lasers. ${ }^{4.5}$ It has been argued that the probable cause of COMD is thermal fusion ${ }^{3}$ or strain ${ }^{5}$ due to local heating resulting from strong absorption of laser light in the vicinity of mirrors or stimulated Brillouin scattering resulting from a strong laser 